\title{
Predictability of early changes in derived neutrophil-to-lymphocyte ratio and neutrophil-to-lymphocyte ratio in patients with advanced non-small cell lung cancer treated with immune checkpoint inhibitors
}

\author{
Jeong Uk Lim ${ }^{1}$, Hye Seon Kang ${ }^{2}$, Chang Dong Yeo ${ }^{3}$, Ju Sang Kim ${ }^{4}$, Chan Kwon Park ${ }^{1}$, Jin Woo Kim ${ }^{5}$, \\ Seung Joon Kim ${ }^{6,7}$, Sang Haak Lee
}

${ }^{1}$ Division of Pulmonary, Critical Care and Allergy, Department of Internal Medicine, Yeouido St. Mary's Hospital, College of Medicine, The Catholic University of Korea, Seoul, Republic of Korea; ${ }^{2}$ Division of Pulmonary, Critical Care and Allergy, Department of Internal Medicine, Bucheon St. Mary's Hospital, College of Medicine, The Catholic University of Korea, Seoul, Republic of Korea; ${ }^{3}$ Division of Pulmonary, Critical Care and Sleep Medicine, Department of Internal Medicine, Eunpyeong St. Mary's Hospital, College of Medicine, The Catholic University of Korea, Seoul, Republic of Korea; ${ }^{4}$ Division of Pulmonary, Critical Care and Sleep Allergy, Department of Internal Medicine, Incheon St. Mary's Hospital, College of Medicine, The Catholic University of Korea, Seoul, Republic of Korea; ${ }^{5}$ Division of Pulmonary, Critical Care and Sleep Medicine, Department of Internal Medicine, Uijeongbu St. Mary's Hospital, College of Medicine, The Catholic University of Korea, Seoul, Republic of Korea; ${ }^{6}$ Division of Pulmonary, Critical Care and Allergy, Department of Internal Medicine, Seoul St. Mary's Hospital, College of Medicine, The Catholic University of Korea, Seoul, Republic of Korea; ${ }^{7}$ Cancer Research Institute, College of Medicine, The Catholic University of Korea, Seoul, Republic of Korea Contributions: (I) Conception and design: JU Lim, CD Yeo; (II) Administrative support: CK Park, SH Lee; (III) Provision of study materials or patients: HS Kang, CD Yeo, JS Kim, CK Park, JW Kim, SJ Kim; (IV) Collection and assembly of data: HS Kang, CD Yeo, JS Kim, CK Park, JW Kim, SJ Kim; (V) Data analysis and interpretation: JU Lim; (VI) Manuscript writing: All authors; (VII) Final approval of manuscript: All authors.

Correspondence to: Chan Kwon Park, MD, PhD. Division of Pulmonology and Critical Care Medicine, Department of Internal Medicine, Yeouido St. Mary`s Hospital, College of Medicine, The Catholic University of Korea, \#62 Yeouido-dong, Yeongdeungpo-gu, Seoul 150-713, Republic of Korea. Email: ckpaul@catholic.ac.kr.

Background: As association between systemic inflammation and disease progression has been suggested, early changes in neutrophil-to-lymphocyte ratio (NLR) and derived NLR (dNLR) may have accurate predictability for prognosis in non-small cell lung cancer (NSCLC) treated with ICI therapy.

Methods: Complete blood count (CBC) was measured immediately before the first and second cycles of ICI therapy in patients with advanced NSCLC. Differences in NLR and dNLR were measured. When the increase in NLR was $\geq 1$, the patient was classified into the increased NLR group. Similarly, when the increase in $\mathrm{dNLR}$ was $\geq 1$, the patient was classified into the increased dNLR group; otherwise, they were classified into the non-increased NLR or dNLR group.

Results: A total of 89 patients was selected for evaluation. Median progression-free survival (PFS) was significantly shorter in the increased NLR group than in the non-increased NLR group (2.6 vs. 9.5 months, $\mathrm{P}<0.001)$. The increased dNLR group showed significantly shorter median PFS than the non-increased dNLR group (4.2 vs. 9.2 months, $\mathrm{P}=0.001$ ). Association with PFS was analyzed using the Cox regression model. In model 1 , increase $\geq 1$ in NLR showed significant association (HR $=3.085,95 \%$ CI, 1.657-5.742, $\mathrm{P}<0.001$ ). In model 2, increase $\geq 1$ in dNLR showed significant association (HR $=2.826,95 \% \mathrm{CI}, 1.436-5.561$, $\mathrm{P}=0.003)$.

Conclusions: Early changes in dNLR were shown to have prognostic value in patients undergoing immunotherapy. It can be an accurate and a comprehensive biomarker for predicting ICI response.

Keywords: Non-small cell lung cancer (NSCLC); immune checkpoint inhibitors; nivolumab 
Submitted Dec 01, 2020. Accepted for publication Mar 05, 2021.

doi: $10.21037 /$ jtd-20-3416

View this article at: http://dx.doi.org/10.21037/jtd-20-3416

\section{Introduction}

Lung cancer is the one of the major causes of cancer-related deaths worldwide $(1,2)$, and non-small cell lung cancer (NSCLC) accounts for $85 \%$ of lung cancers (3). In various types of tumors, the crucial role of programmed death (PD)-1 in tumor-induced immunosuppression has been demonstrated (4). Immune checkpoint inhibitors (ICIs) recently became a main anticancer treatment modality in NSCLC due to understanding of the PD-1/PD-ligand 1 (PD-L1) pathway in tumor-related immune reactions (5-7). Nivolumab, a PD-1 ICI antibody, significantly improved survival in patients previously treated for advanced NSCLC compared with docetaxel $(8,9)$. Pembrolizumab and atezolizumab also showed favorable results $(10,11)$.

Biomarkers that are predictive of treatment responses are essential to determine indication for ICI treatment in NSCLC patients (12). Such parameters with prognostic value will enable pretreatment risk-stratification and an appropriate treatment strategy for an individual patient (13). PD-L1 is the only reliable biomarker used to predict ICI responses $(10,14)$. However, despite favorable results in trials, not all PD-L1-positive NSCLC patients benefit from immunotherapy, with substantial heterogeneity in treatment response and survival (15-17).

Systemic inflammation has been proposed as a mechanism of resistance to antitumor activity in cancer and promotes cancer growth, metastasis, and oncogenic signal activation (18). Furthermore, increased systemic inflammatory biomarkers were reportedly associated with poor outcomes in NSCLC patients (3,19-21).

Neutrophil-to-lymphocyte ratio (NLR) predicts disease progression and overall survival (OS) in NSCLC patients (22-24). Derived NLR (dNLR) was used as an alternative to NLR in studies in which lymphocyte count was not available (25) and was shown to predict poor prognosis in both hematologic and solid tumors (26-29). Although NLR and dNLR were shown to have prognostic value in patients with advanced NSCLC who were receiving ICI $(22,23)$, the biomarkers measured at baseline do not reflect changes in inflammatory status after initiation of chemotherapy. Immune responses and the associated expression in tumor cells and cytokines change after starting ICIs, and these variations may be associated with anticancer treatment response (30-32). Early changes in inflammatory biomarkers are potentially a more accurate predictive tool. The serial change in NLR reportedly showed prognostic value in patients with advanced NSCLC who were taking nivolumab (33). However, dNLR has not been evaluated for early variation after immunotherapy.

In the present study, the predictability of clinical outcomes associated with early changes in NLR and dNLR was assessed in a retrospective cohort of patients with advanced NSCLC administered ICI.

We present the following article in accordance with the STROBE reporting checklist (available at http://dx.doi. org/10.21037/jtd-20-3416).

\section{Methods}

\section{Patient selection}

Study subjects were consecutively selected from a multicenter cohort of lung cancer patients with advanced (stage IIIB-IV) NSCLC who were administered ICI. Patients treated with ICI between January 2016 and December 2019 were enrolled from six university hospitals: Yeouido St. Mary's Hospital, Seoul St. Mary's Hospital, Bucheon St. Mary's Hospital, Eunpyeong St. Mary's Hospital, St. Vincent Hospital, and Uijeongbu St. Mary's Hospital (20). Inclusion criteria for enrollment were patients with (I) records of treatment with nivolumab, pembrolizumab, or atezolizumab; (II) complete blood count (CBC) differential data at the time of initial diagnosis, (III) at least two cycles of ICI treatment, and (IV) all clinical data available from electronic medical records. Exclusion criteria were SCLC, significant infection, or a concurrent hematologic disease (20).

None of the patients enrolled received ICI treatment as first-line treatment due to a government-supervised medical reimbursement policy in Korea.

\section{OS and progression-free survival (PFS)}

Response evaluation was performed following Response Evaluation Criteria in Solid Tumors version 1.1 (34). Patients underwent a computed tomography scan after every two treatment cycles, while treating physicians and 
independent radiologists evaluated the responses. OS was defined as time duration from the date of ICI initiation to death. PFS was defined as the time from ICI initiation to disease progression after first-line treatment. Patients were considered censored when they died or lost contact during the follow-up period (20).

\section{$N L R$ and $d N L R$}

NLR was defined as absolute neutrophil count divided by absolute lymphocyte count. The dNLR was calculated as (total white blood cell count - absolute neutrophil count)/ total white blood cell count.

\section{Inflammatory marker measurement time points}

Complete blood count (CBC) was measured immediately before the first cycle of ICI (within 14 days) and before the second cycle of ICI (within 14 days). Differences in NLR and dNLR were measured, and decrease or increase in markers was recorded.

When the increase in NLR was $\geq 1$, the patient was classified into the increased NLR group; otherwise, they were classified into the non-increased NLR group. Similarly, when the increase in dNLR was $\geq 1$, the patient was classified into the increased dNLR group; otherwise, they were classified into the non-increased dNLR group.

\section{Statistical analysis}

All statistical analyses were performed using the Statistical Package for Social Sciences software version 20.0 (SPSS Inc., Chicago, IL, USA). Data of continuous variables are shown as mean or median with range. The Chi-square test was performed to compare categorical parameters, and continuous variables were compared using two-sided $t$-test or the Mann-Whitney $U$ test depending on whether the variable were normally distributed.

Univariate analysis using the Cox regression model was performed to determine variables significantly associated with OS and PFS. The median OS and PFS are presented as interquartile ranges. Survival curves were constructed using Kaplan-Meier analysis. A log-rank test was performed to determine significant differences in survival outcomes between groups. Statistically significant variables were entered into multivariate analysis using the Cox proportional hazards regression model. A $\mathrm{P}$ value $<0.05$ was considered statistically significant in all analyses.

\section{Ethics statement}

The study was conducted in accordance with the Declaration of Helsinki (as revised in 2013). The present study was approved by the Ethics Committees of Seoul St. Mary's Hospital, Yeouido St. Mary's Hospital, Bucheon St. Mary's Hospital, Eunpyeong St. Mary's Hospital, St. Vincent Hospital, and Uijeongbu St. Mary's Hospital (XC20RIDI0136). The need for informed consent was waived by the Institutional Review Boards.

\section{Results}

\section{Clinical characteristics of study patients}

A total of 89 patients was selected for evaluation. The median age of the patients was 67 years, and 62 (69.7\%) were male. Regarding Eastern Cooperative Oncology Group (ECOG) scale, 85 (95.5\%) patients had ECOG score of 0 or 1 . Adenocarcinoma was pathologically confirmed in $58(65.2 \%)$ patients, and EGFR mutation was found in $9(10.1 \%)$ patients. Regarding smoking history, 68 (76.4\%) patients were ever smokers. Thirty-three (37.1\%) patients were treated with nivolumab, while $56(62.9 \%)$ received pembrolizumab. Sixty-eight (76.4\%) patients had undergone two or more prior chemotherapy lines. Median OS was 15.0 months, and median PFS was 7.7 months. Twenty-one (23.6\%) patients did not respond to ICI treatment (Table 1).

\section{Comparison between groups stratified based on change in $N L R$ or $d N L R$}

Among the 89 patients, 22 showed increase $\geq 1$ in NLR (increased NLR group), while the other $67 \mathrm{did}$ not (nonincreased NLR group). Significant difference was not observed in median age, sex, ECOG, or histologic features. The increased NLR group showed significantly larger proportion of EGFR mutation ( $22.7 \%$ vs. $6.0 \%, \mathrm{P}=0.024)$ compared with the non-increased NLR group. Regarding prior chemotherapy lines, the proportion of patients who received two or more chemotherapy lines was higher in the non-increased NLR group than in the increased NLR group $(76.1 \%$ vs. $54.5 \%)$ but without statistical significance $(\mathrm{P}=0.054)$. Furthermore, the proportion of recent steroid use $(<1$ month prior) before the initiation of ICI did not show statistically significant difference.

Although median OS showed no significant difference between the groups, median PFS was significantly shorter in 
Table 1 Clinical characteristics of study patients

\begin{tabular}{|c|c|}
\hline Parameters & Overall patients \\
\hline Number of patients & 89 \\
\hline Median age, range & $67[46-84]$ \\
\hline \multicolumn{2}{|l|}{ Sex } \\
\hline Male & $62(69.7 \%)$ \\
\hline Female & $27(30.3 \%)$ \\
\hline \multicolumn{2}{|l|}{ ECOG } \\
\hline 0 and 1 & $85(95.5 \%)$ \\
\hline$\geq 2$ & $4(4.5 \%)$ \\
\hline \multicolumn{2}{|l|}{ Histologic features } \\
\hline Adenocarcinoma & $58(65.2 \%)$ \\
\hline Squamous & $28(31.5 \%)$ \\
\hline Adenosquamous & $3(3.4 \%)$ \\
\hline EGFR mutation & $9(10.1 \%)$ \\
\hline Ever smoker & $68(76.4 \%)$ \\
\hline \multicolumn{2}{|l|}{ Immune checkpoint inhibitor } \\
\hline Nivolumab & $33(37.1 \%)$ \\
\hline Pembrolizumab & $56(62.9 \%)$ \\
\hline \multicolumn{2}{|l|}{ Prior chemotherapy (line) } \\
\hline$\geq 2$ & $68(76.4 \%)$ \\
\hline$<2$ & $21(23.6 \%)$ \\
\hline Median OS (months) (IQR) & $15.0(8.4-21.4)$ \\
\hline Median PFS (months) (IQR) & $7.7(2.6-22.3)$ \\
\hline \multicolumn{2}{|l|}{ Best response } \\
\hline Partial response & $29(32.6 \%)$ \\
\hline Stable disease & $38(42.7 \%)$ \\
\hline Progressive disease & $21(23.6 \%)$ \\
\hline NLR & $2.5(0.7-44.0)$ \\
\hline $\mathrm{dNLR}$ & $1.6(0.5-13.7)$ \\
\hline
\end{tabular}

EGFR, epidermal growth factor receptor; ECOG, Eastern Cooperative Oncology Group; IQR, interquartile range; NLR, neutrophil-lymphocyte ratio; dNLR, derived NLR; OS, overall survival; PFS, progression-free survival.

the increased NLR group than in the non-increased NLR group (2.6 vs. 9.5 months, $\mathrm{P}<0.001$; Figure $1 A$ ). Baseline NLR and dNLR and PD-L1 expression at diagnosis showed no significant difference.
When stratified based on change in dNLR, 73 patients were categorized into the non-increased dNLR group, while 16 patients were categorized into the increased dNLR group. Median age, sex, ECOG, histologic features, and smoking history showed no significant difference between groups. A significantly larger proportion of patients in the increased dNLR group showed EGFR mutation (25.0\%) compared to those in the non-increased dNLR group (6.8\%) $(\mathrm{P}=0.029)$. The non-increased $\mathrm{dNLR}$ group showed a significantly larger proportion of patients who received two or more prior chemotherapy lines than did the increased dNLR group ( $75.3 \%$ vs. $50.0 \%, \mathrm{P}=0.044)$. Median OS showed no significant difference; however, the increased dNLR group showed significantly shorter median PFS compared with the non-increased dNLR group (4.2 vs. 9.2 months, $\mathrm{P}=0.001$; Figure $1 B$ ). Baseline NLR and dNLR and PD-L1 expression at diagnosis showed no significant difference (Table 2). In addition, no significant difference in the proportion of recent steroid use was present.

\section{Association with OS and PFS}

Stratification using both NLR and dNLR did not show significant association with OS in univariate analysis. In univariate analysis for association with PFS, age, NLR increase, dNLR increase, and PD-L1 expression showed significant association. In model 1 , in which NLR increase was entered in the multivariate analysis, increase of 1 or more in NLR showed significant association $(\mathrm{HR}=3.085$, 95\% CI, 1.657-5.742, $\mathrm{P}<0.001)$. In model 2 , in which dNLR increase was entered in the multivariate analysis, increase of 1 or more in dNLR showed significant association $(\mathrm{HR}=2.826,95 \% \mathrm{CI}, 1.436-5.561, \mathrm{P}=0.003$; Table 3).

\section{Discussion}

In this multicenter retrospective cohort study, predictability for treatment response of early changes in NLR and dNLR was assessed in patients with advanced NSCLC receiving ICI. Early change of dNLR showed reliable predictability for early disease progression.

Variation in systemic inflammatory status after initiation of ICI has been reported in several studies. Significant changes in serum cytokine concentrations were observed after initiation of ICI in patients with NSCLC. In the study by Boutsikou et al., early changes in IL-6 and IL-8 

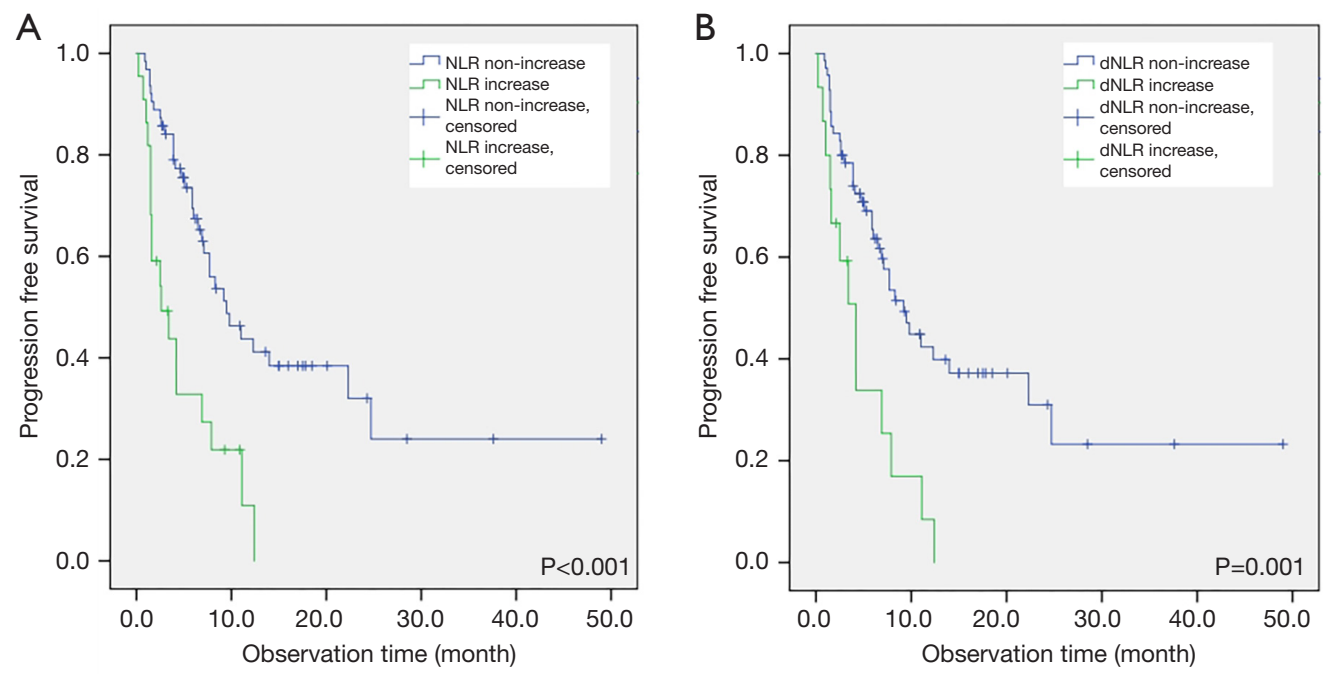

Figure 1 Comparison of median PFS between groups stratified based on (A) change in NLR; (B) change in dNLR. NLR, neutrophillymphocyte ratio; dNLR, derived NLR; PFS, progression-free survival.

were observed and associated with treatment response and survival (32). Sanmamed et al. reported that early change in serum IL-8 level after the start of ICI therapy was associated with treatment response in a study of NSCLC patients treated with nivolumab or pembrolizumab (30). It is assumed that immune responses against tumor cells and associated systemic inflammatory status are not static but dynamic.

ICI has become a mainstay treatment modality for NSCLC, yet, due to relatively shorter clinical treatment experiences, more efforts and studies are necessary for evaluation of biomarkers predicting treatment responses. PD-L1 expression detected based on immunohistochemistry is the most widely used biomarker for identifying candidates with advanced NSCLC who are likely to show favorable response to immunotherapy (35). Other potential biomarkers for response to ICI include tumor mutational burden, which is the total number of mutations, tumorinfiltrating lymphocytes, tumor-specific genotypes, and gene expression signatures from the cancer tissue (36-40). However, tissue-based biomarkers are unfit for serial monitoring, and factors such as sites of biopsy and status of pathologic samples may be bias factors.

NLR and PLR are biomarkers that can be measured in the serum of patients and show correlation with treatment responses to ICI $(24,41)$. Because serum-based biomarkers are available and suitable for multiple measurements, several efforts have been made to increase their predictability. In the present study, early increase of NLR and dNLR showed high predictability for disease progression. Although early change of dNLR was shown to have predictive value in the present study, this biomarker could be used as an adjunct to NLR to assess longitudinal change in inflammatory status associated with tumor cells. This is an inexpensive and easily available biomarker.

The mechanism of the association of increase of dNLR with poor prognosis must be determined. The systemic inflammatory status changed in a relatively short time interval but not in favor of anticancer activity. Neutrophilic inflammation is reportedly associated with metastasis, angiogenesis, and cancer cell proliferation (42-44) and has negative effects on antitumor activities.

When combined with cell-free DNA, an increase in NLR was associated with poor prognosis in a study of patients with NSCLC who were taking nivolumab (33). Similar to NLR, we assumed that early change of dNLR can be used to predict treatment response to ICI, and dNLR may be useful in situations where exact complete blood differential count is not available (45). The serial change of dNLR is significantly more informative than a single measurement. Furthermore, considering that lymphocytes were suggested to have essential roles in antitumor activities $(28,46)$, it is likely that an early increase in dNLR represents inefficacious treatment response after initiation of ICI therapy.

Several limitations are present regarding the study results. First, the small number of study patients is a limitation of the present study. It is possible that clinical 
Table 2 Comparison between clinical characteristics between the groups stratified by the changes in NLR or derived NLR

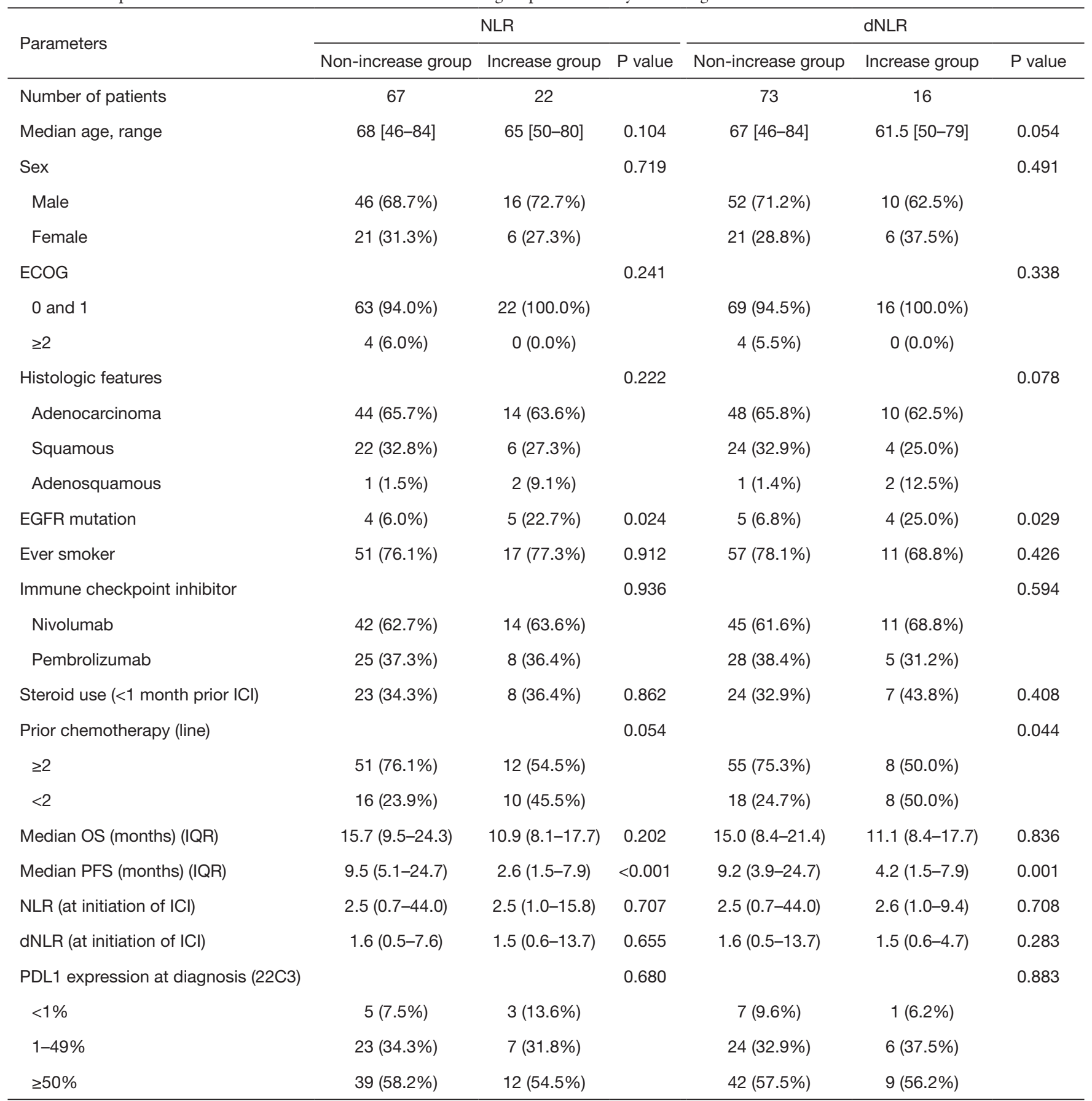

EGFR, epidermal growth factor receptor; ECOG, Eastern Cooperative Oncology Group; ICI, immune checkpoint inhibitor; IQR, interquartile range; NLR, neutrophil-lymphocyte ratio; dNLR, derived NLR; OS, overall survival; PDL1, programmed death-ligand 1; PFS, progression-free survival. 
Table 3 Multivariable analysis for association with progression free survival (Cox-regression hazard model)

\begin{tabular}{|c|c|c|c|c|c|c|c|c|c|}
\hline Parameters & \multicolumn{3}{|c|}{ Univariate analysis } & \multicolumn{3}{|c|}{ Multivariate analysis (Model 1) } & \multicolumn{3}{|c|}{ Multivariate analysis (Model 2) } \\
\hline Sex (male/female) & 1.068 & $0.608-1.877$ & 0.818 & 0.884 & $0.481-1.624$ & 0.692 & 0.969 & $0.527-1.781$ & 0.920 \\
\hline EGFR mutation (wild type/positive) & 0.562 & $0.238-1.327$ & 0.189 & & & & & & \\
\hline Number of metastatic sites $(\geq 3)$ & 1.704 & $0.723-4.015$ & 0.223 & & & & & & \\
\hline $\begin{array}{l}\text { Histopathology } \\
\text { (non-squamous/squamous) }\end{array}$ & 0.825 & $0.462-1.473$ & 0.515 & & & & & & \\
\hline \multicolumn{10}{|l|}{ PDL1 expression (22C3) } \\
\hline$<1 \%$ & 1 & & 0.022 & 1 & & 0.199 & 1 & & 0.137 \\
\hline $1-49 \%$ & 0.349 & $0.134-0.905$ & 0.030 & 0.436 & $0.157-1.206$ & 0.110 & 0.384 & $0.139-1.062$ & 0.065 \\
\hline$\geq 50 \%$ & 0.278 & $0.112-0.691$ & 0.006 & 0.407 & $0.150-1.100$ & 0.076 & 0.378 & $0.140-1.019$ & 0.055 \\
\hline
\end{tabular}

EGFR, epidermal growth factor receptor; ECOG, Eastern Cooperative Oncology Group; ICl, immune checkpoint inhibitor; NLR, neutrophil-lymphocyte ratio; dNLR, derived NLR; PDL1, programmed death-ligand 1.

effects of some parameters would change if the number of study patients was larger. Despite the small sample size, the biomarkers investigated showed significant association. Second, a direct comparison with other reported biomarkers such as baseline NLR was not done in our study due to the limited sample size. Future studies including larger study cohorts are necessary for further validation.

As early change in dNLR was shown to have prognostic value for patients undergoing immunotherapy, it can be used to predict early disease progression in patients with advanced NSCLC receiving ICI treatment.

\section{Acknowledgments}

Funding: None.

\section{Footnote}

Reporting Checklist: The authors have completed the STROBE reporting checklist. Available at http://dx.doi. org/10.21037/jtd-20-3416

Data Sharing Statement: Available at http://dx.doi. org/10.21037/jtd-20-3416

Conflicts of Interest: All authors have completed the ICMJE uniform disclosure form (available at http://dx.doi. org/10.21037/jtd-20-3416). The authors have no conflicts of interest to declare.

Ethical Statement: The authors are accountable for all aspects of the work in ensuring that questions related to the accuracy or integrity of any part of the work are appropriately investigated and resolved. The study was conducted in accordance with the Declaration of Helsinki (as revised in 2013). The present study was approved by the Ethics Committees of Seoul St. Mary's Hospital, Yeouido St. Mary's Hospital, Bucheon St. Mary's Hospital, Eunpyeong St. Mary's Hospital, St. Vincent Hospital, and Uijeongbu St. Mary's Hospital (XC20RIDI0136). The need for informed consent was waived by the Institutional Review Boards.

Open Access Statement: This is an Open Access article distributed in accordance with the Creative Commons Attribution-NonCommercial-NoDerivs 4.0 International 
License (CC BY-NC-ND 4.0), which permits the noncommercial replication and distribution of the article with the strict proviso that no changes or edits are made and the original work is properly cited (including links to both the formal publication through the relevant DOI and the license). See: https://creativecommons.org/licenses/by-nc-nd/4.0/.

\section{References}

1. Postmus PE, Kerr KM, Oudkerk M, et al. Early and locally advanced non-small-cell lung cancer (NSCLC): ESMO Clinical Practice Guidelines for diagnosis, treatment and follow-up. Ann Oncol 2017;28 Suppl 4:iv1-iv21.

2. Ferlay J, Soerjomataram I, Dikshit R, et al. Cancer incidence and mortality worldwide: sources, methods and major patterns in GLOBOCAN 2012. Int J Cancer 2015;136:E359-86.

3. Cedres S, Torrejon D, Martinez A, et al. Neutrophil to lymphocyte ratio (NLR) as an indicator of poor prognosis in stage IV non-small cell lung cancer. Clin Transl Oncol 2012;14:864-9.

4. Brahmer JR, Tykodi SS, Chow LQ, et al. Safety and activity of anti-PD-L1 antibody in patients with advanced cancer. N Engl J Med 2012;366:2455-65.

5. Gandhi L, Rodriguez-Abreu D, Gadgeel S, et al. Pembrolizumab plus Chemotherapy in Metastatic NonSmall-Cell Lung Cancer. N Engl J Med 2018;378:2078-92.

6. Paz-Ares L, Luft A, Vicente D, et al. Pembrolizumab plus Chemotherapy for Squamous Non-Small-Cell Lung Cancer. N Engl J Med 2018;379:2040-51.

7. Socinski MA, Jotte RM, Cappuzzo F, et al. Atezolizumab for First-Line Treatment of Metastatic Nonsquamous NSCLC. N Engl J Med 2018;378:2288-301.

8. Brahmer J, Reckamp KL, Baas P, et al. Nivolumab versus Docetaxel in Advanced Squamous-Cell Non-Small-Cell Lung Cancer. N Engl J Med 2015;373:123-35.

9. Borghaei H, Paz-Ares L, Horn L, et al. Nivolumab versus Docetaxel in Advanced Nonsquamous Non-Small-Cell Lung Cancer. N Engl J Med 2015;373:1627-39.

10. Herbst RS, Baas P, Kim DW, et al. Pembrolizumab versus docetaxel for previously treated, PD-L1-positive, advanced non-small-cell lung cancer (KEYNOTE-010): a randomised controlled trial. Lancet 2016;387:1540-50.

11. Rittmeyer A, Barlesi F, Waterkamp D, et al. Atezolizumab versus docetaxel in patients with previously treated non-small-cell lung cancer (OAK): a phase 3, openlabel, multicentre randomised controlled trial. Lancet 2017;389:255-65.
12. Mezquita L, Auclin E, Ferrara R, et al. Association of the Lung Immune Prognostic Index With Immune Checkpoint Inhibitor Outcomes in Patients With Advanced Non-Small Cell Lung Cancer. JAMA Oncol 2018;4:351-7.

13. Halabi S, Owzar K. The importance of identifying and validating prognostic factors in oncology. Semin Oncol 2010;37:e9-18.

14. Aguiar PN Jr, De Mello RA, Hall P, et al. PD-L1 expression as a predictive biomarker in advanced non-small-cell lung cancer: updated survival data. Immunotherapy 2017;9:499-506.

15. Sharma P, Hu-Lieskovan S, Wargo JA, et al. Primary, Adaptive, and Acquired Resistance to Cancer Immunotherapy. Cell 2017;168:707-23.

16. Garde-Noguera J, Martin-Martorell P, De Julian M, et al. Predictive and prognostic clinical and pathological factors of nivolumab efficacy in non-small-cell lung cancer patients. Clin Transl Oncol 2018;20:1072-9.

17. Park YJ, Kuen DS, Chung Y. Future prospects of immune checkpoint blockade in cancer: from response prediction to overcoming resistance. Exp Mol Med 2018;50:1-13.

18. Hanahan D, Weinberg RA. Hallmarks of cancer: the next generation. Cell 2011;144:646-74.

19. Tomita M, Shimizu T, Ayabe T, et al. Elevated preoperative inflammatory markers based on neutrophilto-lymphocyte ratio and C-reactive protein predict poor survival in resected non-small cell lung cancer. Anticancer Res 2012;32:3535-8.

20. Lim JU, Yeo CD, Kang HS, et al. Prognostic value of platelet count and lymphocyte to monocyte ratio combination in stage IV non-small cell lung cancer with malignant pleural effusion. PLoS One 2018;13:e0200341.

21. Yao Y, Yuan D, Liu H, et al. Pretreatment neutrophil to lymphocyte ratio is associated with response to therapy and prognosis of advanced non-small cell lung cancer patients treated with first-line platinum-based chemotherapy. Cancer Immunol Immunother 2013;62:471-9.

22. Jin J, Yang L, Liu D, et al. Association of the neutrophil to lymphocyte ratio and clinical outcomes in patients with lung cancer receiving immunotherapy: a meta-analysis. BMJ Open 2020;10:e035031.

23. Dusselier M, Deluche E, Delacourt N, et al. Neutrophilto-lymphocyte ratio evolution is an independent predictor of early progression of second-line nivolumab-treated patients with advanced non-small-cell lung cancers. PLoS One 2019;14:e0219060.

24. Nakaya A, Kurata T, Yoshioka H, et al. Neutrophilto-lymphocyte ratio as an early marker of outcomes in 
patients with advanced non-small-cell lung cancer treated with nivolumab. Int J Clin Oncol 2018;23:634-40.

25. Ferrucci PF, Ascierto PA, Pigozzo J, et al. Baseline neutrophils and derived neutrophil-to-lymphocyte ratio: prognostic relevance in metastatic melanoma patients receiving ipilimumab. Ann Oncol 2016;27:732-8.

26. Li Y, Shao Y, Bai L, et al. Increased derived neutrophilto-lymphocyte ratio and Breast Imaging-Reporting and Data System classification predict poor survival in patients with non-distant metastatic HER2+ breast cancer treated with neoadjuvant chemotherapy. Cancer Manag Res 2018;10:3841-7.

27. Wu YY, Qin YY, Qin JQ, et al. Diagnostic value of derived neutrophil-to-lymphocyte ratio in patients with ovarian cancer. J Clin Lab Anal 2019;33:e22833.

28. Zhou X, Sun X, Zhao W, et al. Prognostic significance of peripheral blood absolute lymphocyte count and derived neutrophil to lymphocyte ratio in patients with newly diagnosed extranodal natural killer/T-cell lymphoma. Cancer Manag Res 2019;11:4243-54.

29. Chen L, Kong X, Yan C, et al. The Research Progress on the Prognostic Value of the Common Hematological Parameters in Peripheral Venous Blood in Breast Cancer. Onco Targets Ther 2020;13:1397-412.

30. Sanmamed MF, Perez-Gracia JL, Schalper KA, et al. Changes in serum interleukin-8 (IL-8) levels reflect and predict response to anti-PD-1 treatment in melanoma and non-small-cell lung cancer patients. Ann Oncol 2017;28:1988-95.

31. Ozawa Y, Amano Y, Kanata K, et al. Impact of early inflammatory cytokine elevation after commencement of PD-1 inhibitors to predict efficacy in patients with nonsmall cell lung cancer. Med Oncol 2019;36:33.

32. Boutsikou E, Domvri K, Hardavella G, et al. Tumour necrosis factor, interferon-gamma and interleukins as predictive markers of antiprogrammed cell-death protein-1 treatment in advanced non-small cell lung cancer: a pragmatic approach in clinical practice. Ther Adv Med Oncol 2018;10:1758835918768238.

33. Passiglia F, Galvano A, Castiglia M, et al. Monitoring blood biomarkers to predict nivolumab effectiveness in NSCLC patients. Ther Adv Med Oncol 2019;11:1758835919839928.

34. Eisenhauer EA, Therasse P, Bogaerts J, et al. New response evaluation criteria in solid tumours: revised RECIST guideline (version 1.1). Eur J Cancer 2009;45:228-47.

35. Reck M, Rodriguez-Abreu D, Robinson AG, et al. Pembrolizumab versus Chemotherapy for PD-L1-
Positive Non-Small-Cell Lung Cancer. N Engl J Med 2016;375:1823-33.

36. Yarchoan M, Hopkins A, Jaffee EM. Tumor Mutational Burden and Response Rate to PD-1 Inhibition. N Engl J Med 2017;377:2500-1.

37. Bodor JN, Boumber Y, Borghaei H. Biomarkers for immune checkpoint inhibition in non-small cell lung cancer (NSCLC). Cancer 2020;126:260-70.

38. Hellmann MD, Ciuleanu TE, Pluzanski A, et al. Nivolumab plus Ipilimumab in Lung Cancer with a High Tumor Mutational Burden. N Engl J Med 2018;378:2093-104.

39. Brambilla E, Le Teuff G, Marguet S, et al. Prognostic Effect of Tumor Lymphocytic Infiltration in Resectable NonSmall-Cell Lung Cancer. J Clin Oncol 2016;34:1223-30.

40. Zeng DQ, Yu YF, Ou QY, et al. Prognostic and predictive value of tumor-infiltrating lymphocytes for clinical therapeutic research in patients with non-small cell lung cancer. Oncotarget 2016;7:13765-81.

41. Takeda T, Takeuchi M, Saitoh M, et al. Neutrophil-tolymphocyte ratio after four weeks of nivolumab administration as a predictive marker in patients with pretreated non-smallcell lung cancer. Thorac Cancer 2018;9:1291-9.

42. Carus A, Gurney H, Gebski V, et al. Impact of baseline and nadir neutrophil index in non-small cell lung cancer and ovarian cancer patients: Assessment of chemotherapy for resolution of unfavourable neutrophilia. J Transl Med 2013;11:189.

43. Coussens LM, Werb Z. Inflammation and cancer. Nature 2002;420:860-7.

44. Lim JU, Yeo CD, Kim HW, et al. Pleural Neutrophil-toLymphocyte Ratio May Be Associated With Early Disease Progression in Stage IV Non-small Cell Lung Cancer. In Vivo 2020;34:2179-85.

45. Proctor MJ, McMillan DC, Morrison DS, et al. A derived neutrophil to lymphocyte ratio predicts survival in patients with cancer. Br J Cancer 2012;107:695-9.

46. Gooden MJ, de Bock GH, Leffers N, et al. The prognostic influence of tumour-infiltrating lymphocytes in cancer: a systematic review with meta-analysis. Br J Cancer 2011;105:93-103.

Cite this article as: Lim JU, Kang HS, Yeo CD, Kim JS, Park CK, Kim JW, Kim SJ, Lee SH. Predictability of early changes in derived neutrophil-to-lymphocyte ratio and neutrophil-tolymphocyte ratio in patients with advanced non-small cell lung cancer treated with immune checkpoint inhibitors. J Thorac Dis 2021;13(5):2824-2832. doi: 10.21037/jtd-20-3416 